\title{
Validation of a novel UPLC-HRMS method for human whole-blood cyclosporine and comparison with a CMIA immunoassay
}

\author{
XIAOXUE WANG*, WEI QIN*, WENQIAN CHEN, HUIFANG LIU, \\ DAN ZHANG, XIANGLIN ZHANG and PENGMEI LI \\ Department of Pharmacy, China-Japan Friendship Hospital, Beijing 100029, P.R. China
}

Received July 15, 2020; Accepted November 13, 2020

DOI: $10.3892 /$ etm.2021.9623

\begin{abstract}
Therapeutic drug monitoring is an essential tool when managing the therapeutic use of immunosuppressant cyclosporine A (CsA) in cases with solid organ transplantation. In China, the concentration of CsA is primarily measured using immunoassays. However, existing literature recommends mass spectrometry as the current gold standard for the quantitation of CsA. In the present study, it was attempted to develop a novel application to determine CsA concentrations by using ultra-performance liquid chromatography coupled to high-resolution mass spectrometry (UPLC-HRMS). This technique was then compared with a commercially available chemiluminescent microparticle immunoassay (CMIA) and it was investigated how clinical factors may contribute to quantitation differences between the two methods. An UPLC-Orbitrap-MS method was developed to determine CsA concentrations and this method was validated using guidelines put forward by the Food and Drug Administration from the US. In total, 127 blood samples were acquired from patients undergoing kidney transplantation and analyzed by UPLC-HRMS and CMIA assays. The novel method provided sensitive, accurate and precise results. The mean CsA concentration measured by CMIA was significantly higher than that measured by UPLC-HRMS $(85.70 \pm 48.99$ vs. 67.06 $\pm 34.56 \mathrm{ng} / \mathrm{ml}, \mathrm{P}<0.0001)$. Passing Bablok analysis yielded a slope of 1.34 (95\% CI: 1.22-1.47) and an intercept of -2.54 (95\% CI: -10.29-5.52). A group of samples with a higher metabolic ratio (hydroxylated $\mathrm{CsA} / \mathrm{CsA}>1$ ) exhibited larger discrepancies, while a group of samples taken from patients
\end{abstract}

Correspondence to: Professor Pengmei Li, Department of Pharmacy, China-Japan Friendship Hospital, 2 Yinghuadongjie Street, Chaoyang, Beijing 100029, P.R. China

E-mail: lipengmei@yeah.net

${ }^{*}$ Contributed equally

Key words: cyclosporine, high-resolution mass spectrometry, chemiluminescent microparticle immunoassay, method comparison with a longer post-transplantation time ( $>10$ years) featured narrow $95 \%$ CIs from -15.32 to $65.69 \%$, as determined by Bland-Altman analysis. In summary, a reliable, accurate and rapid UPLC-HRMS method for CsA analysis was successfully developed. The measurement of CsA by the CMIA assay in renal transplant patients should be further evaluated with a specific focus on positive bias.

\section{Introduction}

Cyclosporine A (CsA) is a potent immunosuppressant that has been widely used as a first-line therapeutic to prevent solid organ rejection after transplantation (1). However, determining the appropriate dose of CsA is complicated by narrow therapeutic indices and variability in intra- and inter-individual pharmacokinetics and pharmacodynamics $(2,3)$. Therefore, therapeutic drug monitoring (TDM) is highly recommended. During therapy, CsA levels are maintained within a narrow therapeutic window (4). Consequently, it is vital that the concentrations of CsA are measured in blood samples in an accurate and precise manner.

For several decades, semi-automated and automated immunoassays were the most widely used methodology for determining the levels of CsA in the blood. These methods included chemiluminescent immunoassay (CMIA), ELISA, enzyme multiplied immunoassay and fluorescence polarization immunoassay. However, these methods lack specificity, as they cannot clearly distinguish CsA from its metabolites (5-7); this is a significant problem, particularly because the concentration of CsA metabolites increases over the course of treatment.

Ultra-performance liquid chromatography coupled to high-resolution mass spectrometry (UPLC-HRMS) has already been proven as an important analytical tool for the quantification of drugs (8-11). Although triple quadrupole mass spectrometers are commonly present in clinical laboratories $(12,13)$, there are major advantages to the application of UPLC-HRMS. For instance, UPLC-HRMS has a high mass accuracy (2-5 ppm), a resolving power of 150,000 full width at half maximum (FWHM), provides full scan data and the method is simple to develop. Collectively, these factors make UPLC-HRMS a highly useful and powerful tool for high-performance analyses of drugs and their metabolites $(9,10)$. 
However, only a small number of studies have reported on the application of UPLC-HRMS for the detection of CsA concentrations from a clinical viewpoint $(5,6)$. In the present study, an HRMS-based method for the determination of CsA was developed and validated. The novel method was then compared with a commercial CMIA assay by analyzing 127 samples from renal transplant patients.

\section{Materials and methods}

Sample collection. In total, 127 blood samples were collected from patients who had undergone kidney transplantation at the China-Japan Friendship Hospital (Beijing, China) for TDM of CsA from March 2019 to September 2019. All samples were collected using EDTA as an anti-coagulant. Frozen blood samples were supplied in routine blood collection tubes and stored at $-80^{\circ} \mathrm{C}$ prior to analysis.

Materials and reagents. CsA (99\% purity) was purchased from The European Directorate for the Quality of Medicines \& Healthcare and CsA-d4 (95\% purity) was purchased from Toronto Research Chemicals. Chromatographic grade acetonitrile, methanol, ammonium acetate and formic acid, were obtained from Thermo Fisher Scientific, Inc. Zinc sulfate heptahydrate (analytical grade) was obtained from Sinopharm Chemical Reagent Co., Ltd. Deionized water was purified with a Milli-Q Plus Ultrapure water system (Millipore Corp.). The CMIA method was performed on an Abbott ARCHITECT i1000SR system (Abbott Laboratories) and required a calibrator and CsA kit (cat. no. 1L75-25); these were also obtained from Abbott Laboratories. The acceptable ranges of CMIA quality control samples were defined as per the manufacturer's instructions.

Instrumentation and UPLC-HRMS conditions. Experiments were performed on an Ultimate 3000 system combined with a Q Exactive mass spectrometer (Thermo Fisher Scientific, Inc.) that was used in full scan mode and positive electrospray ionization. CsA, hydroxylated $(\mathrm{H}) \mathrm{CsA}$ as the major metabolite and internal standard CsA-d4 [internal standard (ISTD) for CsA] in whole blood samples were separated on an Acquity UPLC BEH C18 column (50x2.1 mm, $1.7 \mu \mathrm{m}$ particle size; Waters) maintained at $40^{\circ} \mathrm{C}$. Mobile phase A consisted of $2 \mathrm{mM}$ ammonium acetate with $0.1 \%$ formic acid (v/v) in water. Mobile phase B consisted of $0.1 \%$ formic acid (v/v) in acetonitrile. The gradient program started with $45 \% \mathrm{~B}$, increasing to $50 \% \mathrm{~B}$ at $1.0 \mathrm{~min}$; this changed to $80 \% \mathrm{~B}$ at $2.5 \mathrm{~min}$. At $3.0 \mathrm{~min}$, the mobile phase increased to $100 \% \mathrm{~B}$ then, hold at $100 \% \mathrm{~B}$ for $2 \mathrm{~min}$. The total instrumental analysis time was $6 \mathrm{~min}$, including re-equilibration of the column. The flow rate was set at $0.3 \mathrm{ml} / \mathrm{min}$ and the temperature of the auto-sampler was set to $10^{\circ} \mathrm{C}$.

The optimized parameters of the HRMS system were as follows: Electron spray ionization positive mode; full MS scan; sheath gas and auxiliary gas flow rates of 28 arbitrary units (AU) and $8 \mathrm{AU}$, respectively; a spray voltage of $3.70 \mathrm{kV}$; a capillary gas heater temperature of $320^{\circ} \mathrm{C}$; a mass to charge ratio $(\mathrm{m} / \mathrm{z})$ range of $1,200-1,220$; and a resolution of 70,000 (FWHM). The maximum injection time was $50 \mathrm{msec}$ and the mass tolerance was set to $5 \mathrm{ppm}$. Analytes and internal standards with respective $\mathrm{m} / \mathrm{z}$ were as follows: $\mathrm{m} / \mathrm{z} 1,202.8485$ for $\mathrm{CsA}[\mathrm{M}+\mathrm{H}]^{+}, \mathrm{m} / \mathrm{z} 1,218.8420$ for $\mathrm{HCsA}[\mathrm{M}+\mathrm{H}]^{+}$and $\mathrm{m} / \mathrm{z} 1,206.8727$ for ISTD $[\mathrm{M}+\mathrm{H}]^{+}$.

Preparation of stock solutions, calibration curves and quality control samples. A stock solution of CsA and a working standard of ISTD were prepared in methanol at concentrations of $200 \mu \mathrm{g} / \mathrm{ml}$ and $400 \mathrm{ng} / \mathrm{ml}$ at room temperature (RT), respectively. Further calibration standards and quality controls (QCs) were prepared by serial dilutions using methanol. A calibration curve for blood CsA was established by diluting the compound in drug-free human blood from different healthy donors to final concentrations of 5, 12.5, 25, 50, 100, 200, 400 and $800 \mathrm{ng} / \mathrm{ml}$, and by preparing QC samples at 15,350 and $700 \mathrm{ng} / \mathrm{ml}$. These solutions were stored in $200-\mu \mathrm{l}$ aliquots at $-80^{\circ} \mathrm{C}$ prior to use.

Sample preparation. Prior to analysis, zinc sulfate heptahydrate solution (200 $\mu 1,400 \mathrm{mM})$ was added to each blood sample, followed by gentle mixing. Subsequently, ISTD working solution $(50 \mu \mathrm{l})$ was added and the mixture was vortexed for $1 \mathrm{~min}$. Afterwards, $400 \mu \mathrm{l}$ of acetonitrile was added and the mixture was vortexed again for $5 \mathrm{~min}$ and then centrifuged at $11,290 \mathrm{x} \mathrm{g}$ for $5 \mathrm{~min}$ at $4^{\circ} \mathrm{C}$. Next, $800 \mu \mathrm{l}$ of supernatant was transferred to a clean polypropylene tube and then evaporated to dryness at $40^{\circ} \mathrm{C}$ in a vacuum centrifugal concentrator. The residues were finally reconstituted with $200 \mu \mathrm{l}$ of the mobile phase A/B (55:45, v/v); 5- $\mu$ l aliquots were injected into the UPLC system for analysis.

\section{Validation of the LC-HRMS assay.}

General. The experimental method was validated in strict accordance with the current guidelines from the Food and Drug Administration (FDA) (14). The validation of the method involved specificity, linearity, the lower limit of quantification (LLOQ), carryover effect, between- and within-run accuracy and precision, matrix effect, recovery and storage stability.

Specificity. Six drug- and internal standard (ISTD)-free blood samples were individually analyzed to detect interference from endogenous components. The specificity was acceptable when the interfering peak area was $<20 \%$ of the peak area of CsA for the LLOQ and 5\% for the ISTD.

Linearity and LLOQ. Linearity was assessed by measuring the seven calibration standard concentrations in the blood. A calibration curve was then created by plotting the peak area ratios of CsA to the ISTD against the concentration. The linearity of the calibration curve was assessed using the weighted least-squares method with the reciprocal of the concentration squared $\left(1 / \mathrm{x}^{2}\right)$ serving as a weighting factor. The LLOQ was evaluated by analyzing five replicates of mixed whole blood samples at a concentration of $5.0 \mathrm{ng} / \mathrm{ml}$. The concentration of $\mathrm{HCsA}$ was then calculated using the CsA calibration curve.

Carryover. Carryover was investigated by injecting five blank samples from different donors after the highest calibration standard $(800 \mathrm{ng} / \mathrm{ml})$. The peak area in the first blank sample was required to be $<20 \%$ of the LLOQ for CsA and $5 \%$ for ISTD. 
Accuracy and precision. The accuracy and precision of the method were repeatedly assessed using four concentrations of QC samples (LLOQ, 15, 350 and $700 \mathrm{ng} / \mathrm{ml}$ ) five times within a single run (intra-day) and in a single series per day on three consecutive days (inter-day). For the accuracy assay, the mean value was required to be within $15 \%$ ( $20 \%$ for LLOQ) of the nominal values for the QC samples. The precision was not supposed to exceed $15 \%$ (20\% for the LLOQ) of the coefficient of variation.

Dilution integrity. To test dilution integrity, five blank matrix samples were spiked with a CsA concentration that was $\sim 50 \%$ above the highest calibration standard and then diluted 5-fold with blank matrix after sample preparation. The measured concentration was then back-calculated and compared to the nominal concentration. The accuracy were required to be within 85 and $115 \%$, the precision should not exceed $\pm 15 \%$, respectively.

Recovery. To evaluate the recovery of CsA, three concentrations of CsA $(15,350$ and $700 \mathrm{ng} / \mathrm{ml})$ were tested using six different blank blood samples. Recovery was then calculated as the mean ratio between the peak area of spiked samples after extraction and prior to extraction (CsA spiked in post-protein precipitated drug-free whole blood). The variation in recovery across all concentrations was required to be consistent and $<15 \%$.

Matrix effect. The matrix factor (MF) was calculated by comparing the peak areas of CsA in blank matrix from six individual donors after sample processing, with the peak areas of CsA in the absence of a matrix composed of methanol and water (50:50, v/v) for three different concentrations of CsA (15, 350 and $700 \mathrm{ng} / \mathrm{ml}$ ). The ISTD-normalized MF (the MF of CsA divided by the MF of $\mathrm{CsA}-\mathrm{d} 4$ ) was required to have a variation of $<15 \%$.

Stability. Stability was tested in triplicate using QC samples $(15,350$ and $700 \mathrm{ng} / \mathrm{ml})$ under different conditions (in whole blood: RT for $12 \mathrm{~h}$, three consecutive freeze-thaw cycles from $-80^{\circ} \mathrm{C}$ to $\mathrm{RT}, 4^{\circ} \mathrm{C}$ for 3 days and $-80^{\circ} \mathrm{C}$ for 60 days; post-treatment: $10^{\circ} \mathrm{C}$ for $24 \mathrm{~h}$ ). A fresh calibration curve was used for each quantification. CsA was assumed to be stable if the mean concentration changes varied within an acceptable range of $\pm 15 \%$ (\%bias) from the nominal concentration.

CMIA assay. The CMIA assay was performed in accordance with the manufacturer's guidelines for the CsA assay kit and was carried out with an automatic ARCHITECT i1000 analyzer (Abbott Park) that was approved by the FDA. For automated analysis, 200- $\mu 1$ aliquots of the samples, calibrator and QCs were introduced into the system. For calibration, a blank calibrator and five multi-level whole-blood calibrators containing concentrations of CsA of 40-1,500 ng/ml, were provided by the manufacturer.

Comparative analyses between UPLC-HRMS and CMIA. In total, 127 CsA samples were analyzed by the UPLC-HRMS method and the CMIA. Normal distributions were evaluated using the Shapiro-Francia test. The correlation coefficients and regression equations of the measurements for the two methods were evaluated by Spearman's test and Passing-Bablok regression (15) using MedCalc version 15 (MedCalc Software bvba) and SPSS version 22 (IBM Corp.). The level of agreement between the two methods was evaluated by Bland-Altman plots (16). The Wilcoxon test in MedCalc was applied to compare the medians obtained from HRMS and CMIA. $\mathrm{P}<0.05$ was considered to indicate a statistically significant difference.

\section{Results}

Validation of the LC-HRMS assay.

General. Representative chromatograms of the LC-HRMS method for CsA, the ISTD and HCsA, are presented in Fig. 1. The analytical run time was 6 min, including the time required to equilibrate the column to baseline conditions prior to the next injection.

Specificity. The LC-HRMS assay had excellent specificity. For the endogenous compounds in the six individual donors of the blank matrix, no evidence of interference was observed.

Linearity and LLOQ. The calibration curve was calculated using 8 calibrators with a linear regression model and weighting factor $\left(1 / x^{2}\right)$. Results were linear $\left(r^{2}>0.993\right)$ within a detection range of 5-800 $\mathrm{ng} / \mathrm{ml}\left(\mathrm{r}^{2}>0.993\right)$. For LLOQ samples, the signal-to-noise ratio was at least $\geq 103$.9.

Carryover. Peak areas for five different drug-free blood samples, as measured after the highest calibrator sample, were consistently below $5.3 \%$ of the LLOQ and below $0.4 \%$ for the ISTD, respectively.

Accuracy and precision. For CsA, the within-run accuracy ranged from 98.6 to $114.9 \%$ (mean, $105.4 \%$ ), the within-run precision ranged from 2.3 to $5.6 \%$, the between-run accuracy ranged from 95.3 to $114.3 \%$ (mean, $103.2 \%$ ) and the between-run precision ranged from 4.7 to $9.2 \%$ at all QC levels. The results for accuracy and precision are summarized in Table I.

Dilution integrity. For diluted samples, the accuracy ranged from 90.4 to $113.1 \%$ and the precision ranged from 1.1 to $4.1 \%$ (Table SI). These data indicated that the mean dilution did not affect the accuracy and precision of the method.

Recovery. The recovery at three QC levels (15, 350 and $700 \mathrm{ng} / \mathrm{ml}$ ) after preparation varied between 86.8 and $92.5 \%$.

Matrix effect. The MFs across all QC levels ranged from 108.3 to $125.4 \%$, and the variation of the ISTD-normalized MF was $<5.5 \%$. These data indicated that when used as the ISTD, $\mathrm{CsA}-\mathrm{d}_{4}$ fully compensated for the matrix effect.

Stability. CsA was stable under all of the conditions tested at three QC levels with an inaccuracy range of -8.2 to $5.8 \%$. These results are presented in Table I.

CMIA assay. The CMIA assay was based on a fully automated method and was performed in accordance with the manufacturer's instructions. The assay was validated at six levels with a range of calibrators at concentrations of $0,40.0,150.0,400.0$, 
Table I. Summary of accuracy, precision and stability of cyclosporine A in human blood using ultra-performance liquid chromatography high-resolution mass spectrometry.

Storage time ${ }^{\mathrm{a}}$

\begin{tabular}{|c|c|c|c|c|c|c|c|c|c|c|}
\hline \multirow[b]{3}{*}{$\begin{array}{l}\text { Compound } \\
\text { (concentration) }\end{array}$} & \multirow{2}{*}{\multicolumn{2}{|c|}{ Intraday }} & \multirow{2}{*}{\multicolumn{2}{|c|}{ Interday }} & \multicolumn{6}{|c|}{ Storage time ${ }^{a}$} \\
\hline & & & & & \multirow[b]{2}{*}{$\begin{array}{l}\mathrm{RT} / \\
12 \mathrm{~h}\end{array}$} & \multirow{2}{*}{$\begin{array}{c}-80^{\circ} \mathrm{C} / 1 \\
\text { freeze-thaw } \\
\text { cycle }\end{array}$} & \multirow{2}{*}{$\begin{array}{c}-80^{\circ} \mathrm{C} / 2 \\
\text { freeze-thaw } \\
\text { cycles }\end{array}$} & \multirow[b]{2}{*}{$\begin{array}{r}4^{\circ} \mathrm{C} / 3 \\
\text { days }\end{array}$} & \multirow[b]{2}{*}{$\begin{array}{c}-80^{\circ} \mathrm{C} / 60 \\
\text { days }\end{array}$} & \multirow{2}{*}{$\begin{array}{c}10^{\circ} \mathrm{C} / 24 \mathrm{~h} \\
\text { (extracted } \\
\text { samples) }\end{array}$} \\
\hline & $\begin{array}{l}\text { Accuracy } \\
(\%)\end{array}$ & $\begin{array}{l}\text { Precision } \\
\text { (RSD, \%) }\end{array}$ & $\begin{array}{c}\text { Accuracy } \\
(\%)\end{array}$ & $\begin{array}{l}\text { Precision } \\
\text { (RSD, \%) }\end{array}$ & & & & & & \\
\hline LLOQ (5 ng/ml) & 102.7 & 2.4 & 114.3 & 5.4 & & & & & & \\
\hline $\mathrm{QC} 1(15 \mathrm{ng} / \mathrm{ml})$ & 114.9 & 5.6 & 110.4 & 9.2 & 3.5 & -3.8 & -4.2 & -6.4 & -4.0 & 0.1 \\
\hline $\mathrm{QC} 2(350 \mathrm{ng} / \mathrm{ml})$ & 105.3 & 3.1 & 97.8 & 4.7 & -5.1 & 2.9 & 2.6 & -8.2 & -3.3 & -5.7 \\
\hline QC3 $(700 \mathrm{ng} / \mathrm{ml})$ & 98.6 & 2.3 & 95.3 & 6.2 & -2.7 & -4.9 & 3.2 & -7.1 & 5.8 & -7.5 \\
\hline
\end{tabular}

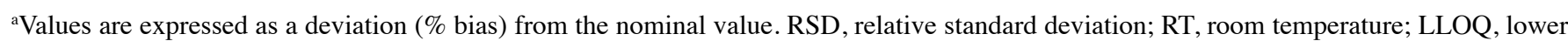
limit of quantification; QC, quality control.
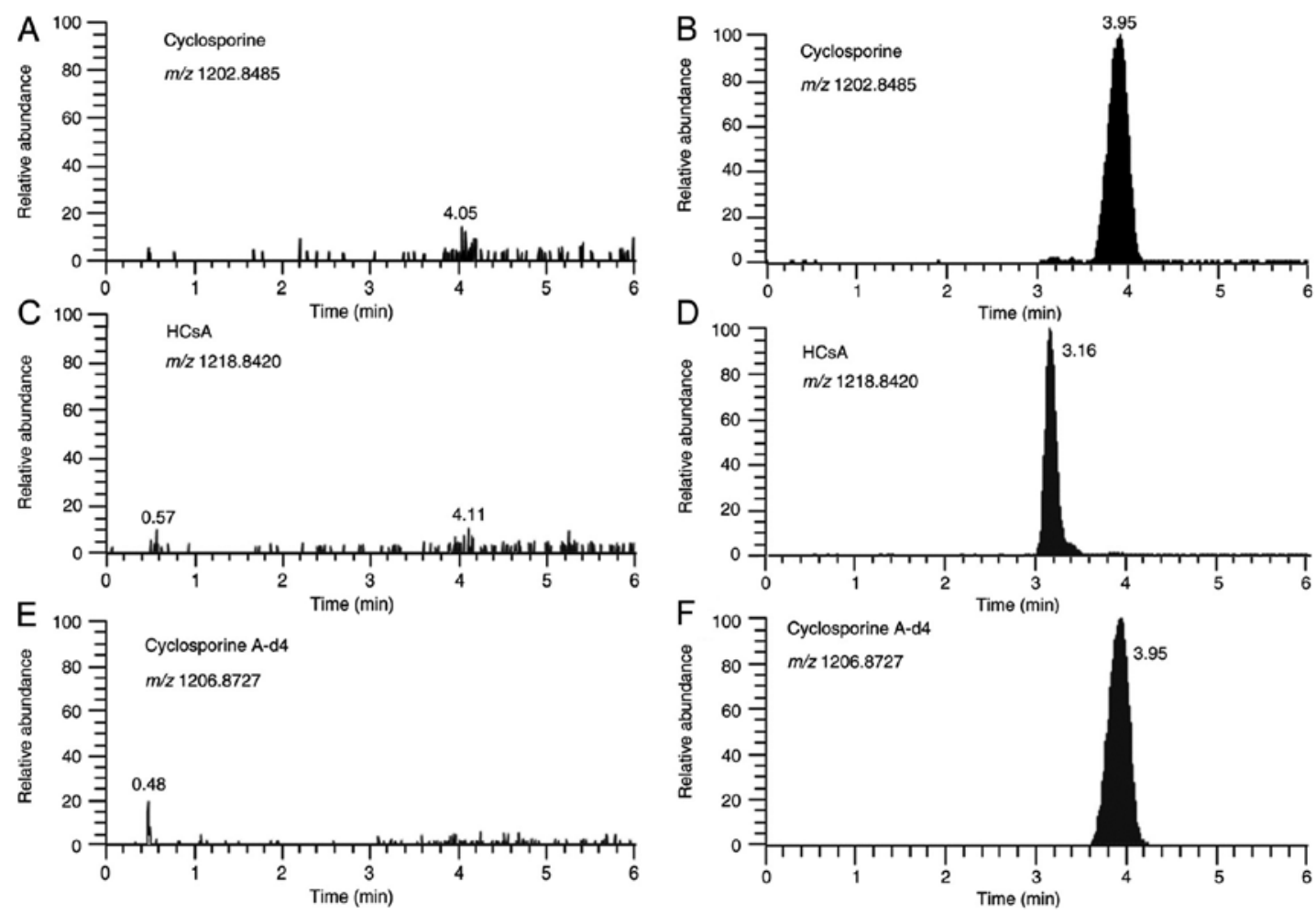

Figure 1. Representative extracted ion chromatograms of cyclosporine. The main metabolites of HCsA and CsA-d4 (internal standard). (A) Extracted ion chromatography of $m / z 1202.8485$ in blank human whole blood. (B) Blank human whole blood spiked with cyclosporine. (C) Extracted ion chromatography of $m / z 1218.8420$ in blank human whole blood. (D) Cyclosporine samples from a representative patient in the HCsA/CsA $>1$ group. (E) Extracted ion chromatography of $m / z$. 1206.8727 in blank human whole blood. (F) Blank human whole blood spiked with an internal standard. m/z, mass to charge ratio; HCsA, hydroxylated cyclosporine A.

800.0 and $1,500.0 \mathrm{ng} / \mathrm{ml}$. To control the quality of the analyses, three levels of QC concentrations were tested with acceptable results. Theresults were $70.3 \mathrm{ng} / \mathrm{ml}$ (target, $79.7 \mathrm{ng} / \mathrm{ml}$; acceptable range, $44.4-115 \mathrm{ng} / \mathrm{ml}), 261.9 \mathrm{ng} / \mathrm{ml}$ (target, $283 \mathrm{ng} / \mathrm{ml}$; acceptable range, $227-340 \mathrm{ng} / \mathrm{ml}$ ) and $812 \mathrm{ng} / \mathrm{ml}(\operatorname{target}, 830 \mathrm{ng} / \mathrm{ml}$; acceptable range, $556-1,105 \mathrm{ng} / \mathrm{ml})$.

Comparison between UPLC-HRMS and CMIA. A total of 127 blood samples were acquired from Chinese renal transplant patients (72 males and 55 females); median age, 51 years (range, 24-76 years). The median number of years post-transplantation was 11 (range, $0.85-24$ ). None of the data were distributed normally $(\mathrm{P}<0.001$, Shapiro-Francia test). The concentration measured by UPLC-HRMS was $67.06 \mathrm{ng} / \mathrm{ml}$ (interquartile range, $50.54-90.41 \mathrm{ng} / \mathrm{ml}$ ); this was significantly lower than that measured by CMIA [ $85.70 \mathrm{ng} / \mathrm{ml}$ (interquartile range, $61.98-115.70 \mathrm{ng} / \mathrm{ml})]$. According to a paired-samples Wilcoxon test, the mean values of these measurements were significantly different $(\mathrm{P}<0.0001)$. Passing-Bablok regression analysis revealed a poor linear correlation between UPLC-HRMS and CMIA (Fig. 2). The regression equation for the assay according to the two methods was $\mathrm{C}_{\mathrm{CMIA}}=-2.54+1.34$ $\mathrm{C}_{\text {UPLC-HRMS }}$ with $\mathrm{r}=0.818$; the $95 \%$ confidence interval for the intercept was -10.29-5.52 (including zero) and the slope 

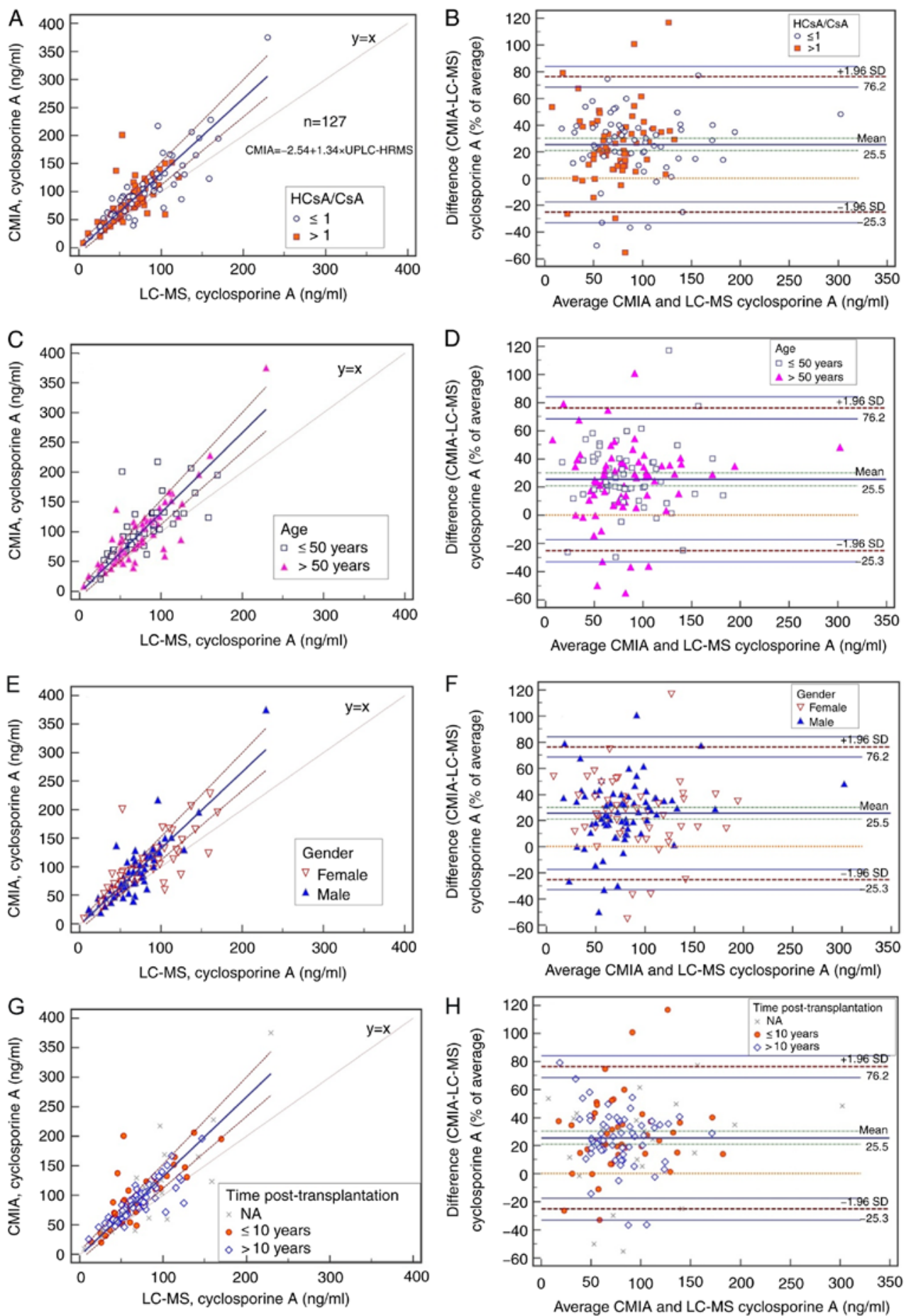

Figure 2. Method comparison using Passing-Bablok regression (left: A, C, E and G) and relative (\%) Bland-Altman analysis (right: B, D, F and H) of the new Ultra-Performance LC High-Resolution MS method vs. the CMIA assay. Sample points for each subgroup include (A and B) metabolic ratio, (C and D) age, (E and F) gender and $(\mathrm{G}$ and $\mathrm{H})$ the length of time after transplantation. The lines of correlation are represented by solid blue lines, and the $95 \%$ limits of agreement are represented by dashed lines. NA, information not available; HCsA, hydroxylated cyclosporine A; SD, standard deviation; LC-MS, liquid chromatography tandem mass spectrometry; CMIA, chemiluminescent microparticle immunoassay.

was 1.22-1.47 (not including 1). As MS is more specific than immunoassay, it is recommended as a gold standard for CsA analysis $(4,17,18)$; thus, UPLC-HRMS was used as the reference method. Considering UPLC-HRMS as the reference method, these results indicated the presence of proportional bias in the CMIA. According to Bland-Altman plots (Fig. 2), a median bias of $25.46 \%$ (range, -25.25 to $76.17 \%$ ) was detected. The highest discrepancy was observed at moderate concentrations.
The International Association for Therapeutic Drug Monitoring and Clinical Toxicology (IATDMCT) has advised that a comparison between two methods should include samples from a wide variety of pathologic conditions (4) and certain reports have indicated that the type of organ transplanted, time after transplantation and time of blood testing (peak or trough levels) may influence the metabolite-to-CsA ratio (19-21). Thus, the patients were separated 

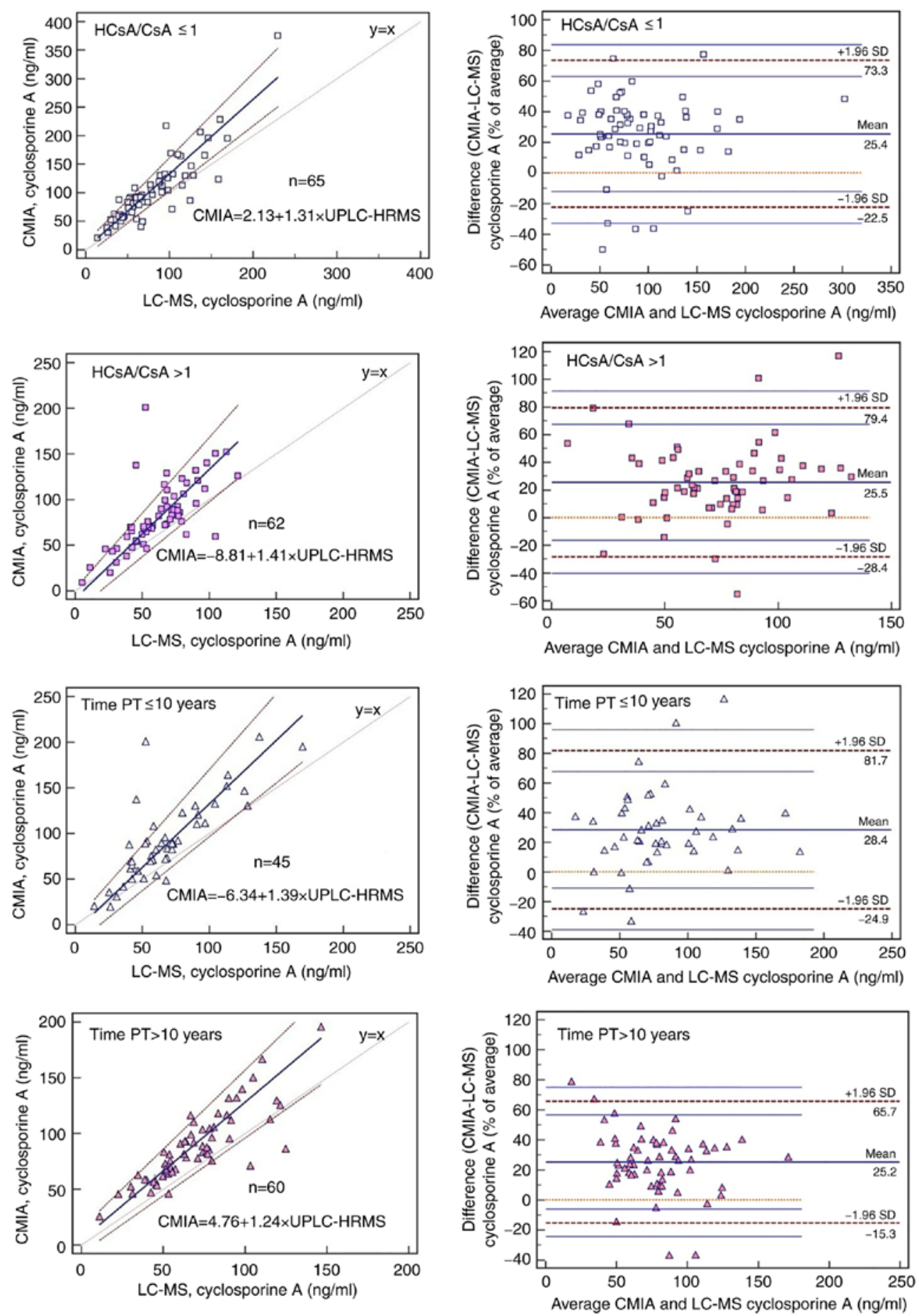

Figure 3. Method comparison using Passing-Bablok regression (left) and relative (\%) Bland-Altman analysis (right) of the new UPLC-HRMS method vs. the CMIA assay. The liner correlation graphs are represented by solid blue lines and the 95\% limits of agreement are represented by dashed lines. PT, post-transplantation; HCsA, hydroxylated cyclosporine A; SD, standard deviation; LC-MS, liquid chromatography tandem mass spectrometry; CMIA, chemiluminescent microparticle immunoassay; UPLC-HRMS, ultra-performance LC high-resolution MS.

into several groups to explore the effect of the metabolic ratio for $\mathrm{CsA}$ (HCsA/CsA) (3), age, gender, and the length of time post-transplantation, on the bias between CMIA and HRMS. In the present study, the sub-groups were designed according to the median in each group (except gender), in order to meet the statistical requirements. As presented in Figs. 2A and 3, when the data were divided according to the ratio of $\mathrm{HCsA}$ to $\mathrm{CsA}$, the highest discrepancy was observed at high ratios $(\mathrm{HCsA} / \mathrm{CsA}>1)$. Table II presents data relating to the detection of CsA by UPLC-HRMS vs. CMIA for the blood samples analyzed and provides detailed results for the Passing-Bablok correlation, Spearman correlation coefficient, a comparison of the correlation coefficients and Bland-Altman mean bias. The concentrations obtained with the two methods differed significantly among all subgroups $(\mathrm{P}<0.0001)$ and higher median values were obtained with CMIA. Although there were no significant differences between subgroups, the HCsA/CsA groups had the lowest 


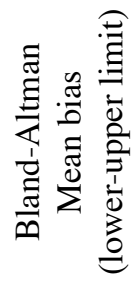

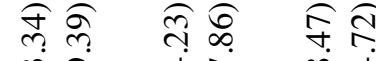

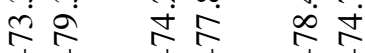

กิ

तิ

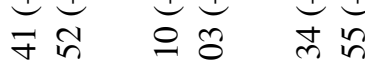

में ते

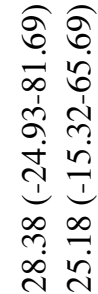

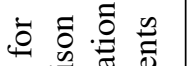

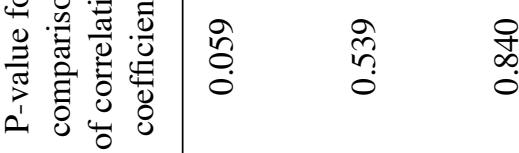

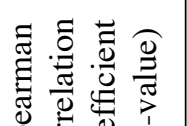

สิ) $\widehat{\bar{\sigma}} \widehat{\bar{\sigma}} \widehat{\bar{\sigma}}$

की :

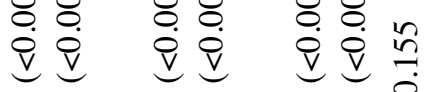

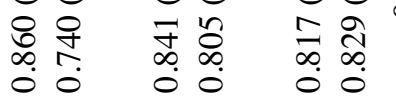

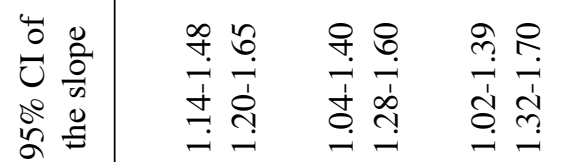

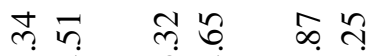

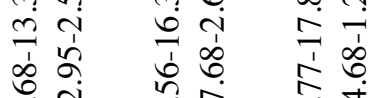

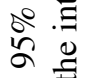

बิ่

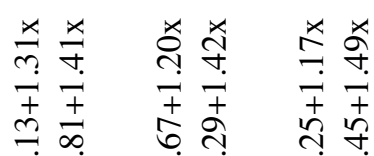

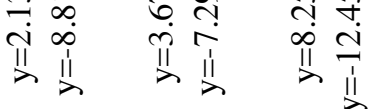

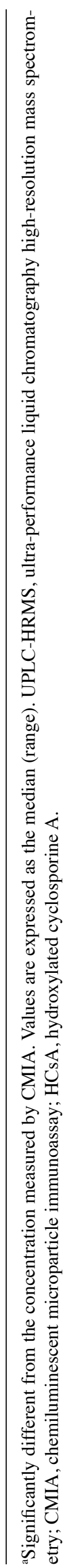


correlation coefficients $(\mathrm{P}=0.059)$ (Table II). When data were grouped according to the length of time after transplantation, the narrowest limits of agreement for mean bias (from -15.32 to $65.69 \%$ ) were observed for the longest post-transplantation time ( $>10$ years), as determined by Bland-Altman analysis (Fig. 3).

\section{Discussion}

In the present study, the successful development of an UPLC-HRMS-orbitrap-based method for the determination of CsA in blood samples was described. This novel method was validated and compared with the commercial CMIA in blood samples from 127 patients. To the best of our knowledge, there is only one other published application of the orbitrap mass spectrometer related to TDM of CsA (5).

Although most published MS methods for immunosuppressants are relying on ammonium adducts of those drugs $(5,6)$. In the present study, the ammonium adduct of CsA had almost the same intensity as the protonated adduct. Furthermore, when the ammonium adduct was chosen as the monitoring ion, the interference peak, i.e. the isotopic peak of $[\mathrm{HCsA}+\mathrm{H}]^{+}$, in patients' whole blood appeared at a retention time of $3.16 \mathrm{~min}$. As the major CsA metabolites are the monohydroxylated AM1 and AM9 and the demethylated AM4N (3), the amount of hydroxylated and methylated metabolite of CsA in-source fragmentation ions $(\mathrm{m} / \mathrm{z}=1,218.8)$ is too small to interfere with the protonated adduct of HCsA. Thus, the present MS method was relying on protonated adduct.

HRMS has numerous advantages over existing methods, including high mass accuracy, high resolution and the ability to analyze data retrospectively (22). Perhaps the only disadvantage of HRMS when compared to the tandem mass spectrometer is the low sensitivity of the quantitative performance. To improve the sensitivity of this method, sample condensation was chosen instead of single organic solvent precipitation. Furthermore, the LLOQ was $5 \mathrm{ng} / \mathrm{ml}$; this was lower than that reported by other HRMS studies $(24.2-28 \mathrm{ng} / \mathrm{ml})(5,6)$, meaning higher sensitivity. The recoveries were 86.8 to $92.5 \%$; these values were similar to those published in other studies (75.7 to $112.0 \%$ ) using the one-step preparation procedure (23-26). The variation of ISTD-normalized MF was $<5.5 \%$; this was lower than the values estimated by previous studies (6.3 to $10.3 \%)(23,24)$. CsA was stable in human whole blood under the tested conditions, which were similar to the conditions used in previous studies (23-26).

The comparison of UPLC-HRMS with CMIA was performed using a large number of samples $(n=127)$, thus allowing for meaningful statistical analysis $(40 \leq n \leq 100)$, as recommended by IATDMCT. In addition, the two methods were compared by an unbiased procedure as Passing Bablok regression and Bland-Altman plot according to the recommendations of the IATDMCT (4). A significant discrepancy was observed between the measurements provided by the two assays. The mean concentration measured by CMIA was higher than that determined by the novel UPLC-Orbitrap-MS based method. On average, the overestimation by CMIA was $\sim 25.5 \%$, but in certain cases, it was as high as $120 \%$.

A well-known problem when quantifying the concentration of CsA with CMIA is interference from CsA metabolites; HCsA (including AM1, AM1c, AM9 and AM99N) may result in $6.9 \%$ cross-reactivity relative to the parent drug. AM1, AM1c and AM9 possess partial immunosuppressive activity; on the other hand, AM1 and AM9 are related to renal transplantation rejection and exhibit nephrotoxic effects $(27,28)$. Although numerous researchers have reported the overestimation of immunoassays with regards to CsA analysis when compared with MS (23-26,29-32), the potential influence of drug-to-metabolite ratios on positive bias has remained to be determined. In the present study, semiquantitative determination of the metabolites HCsA was performed by comparing the integration of the peaks, from HCsA to the area of CsA according to the calculation method of a previous study (11), as standards for the metabolites were not available. Furthermore, AM1, AM1c, AM9 and AM99N were not quantified due to the lack of purified metabolites. The data were then divided into two subgroups by HCsA/CsA. Although the measurements of UPLC-HRMS and CMIA were weakly correlated in the $\mathrm{HCsA} / \mathrm{CsA}>1$ group, overestimation was high in both of the HCsA/CsA groups. As the cross-reactivity of the metabolites HCsA alone was not able to fully explain the overestimation, between-patient variables were considered in the further analysis.

Therefore, a number of between-patient variables, including age, gender and the length of time after transplantation, were analyzed by appropriate statistical methods. The CsA concentration in patients with long post-transplantation times (>10 years) determined by CMIA exhibited the lowest deviation from the UPLC-HRMS results among the parameters in the different patient groups. Higher age did not significantly influence the deviation between the two methods, nor did gender. It appeared that the overestimation of the CMIA when compared to the UPLC-HRMS method may be due to the CsA metabolic ratio and the length of time post-transplantation. The higher levels determined by CMIA are certainly due to the extensive metabolism of CsA and cross-reactivity of the metabolites that cannot be separated from CsA (6,7). However, the influence of the factor of time post-transplantation on the metabolic ratio of CsA remains to be determined. In the present cohort, no further details were available to better investigate this point. It is essential to be able to determine the true value of CsA when managing patients as this result may influence clinical decisions, such as the adjustment of dosage that may lead to viral infection or rejection. Clearly, the use of UPLC-HRMS procedures for TDM of cyclosporine provides a more sensitive means of detection in samples from patients receiving low-dose cyclosporine. This method allows for more selective quantification of samples from new transplant recipients and significantly reduces any errors in dosing that may occur due to metabolite cross-reactivity when combined with varying degrees of overestimation by CMIA.

There are certain deficiencies of the present study: i) Sample preparation was time-consuming; thus, a more convenient method, such as a dephospholipid precipitation plate, will be used in the next study to improve the procedure; ii) the mass range should be assigned as m/z 1,200-1,241 to check the intensity of sodium adducts and rule out a decrease in sensitivity; iii) the influence of co-medicated drugs on CsA analysis were not evaluated.

Ongoing research is now investigating the potential influence of a wide variety of pathological conditions on the observed bias between these two methods, including different transplant types, ethnic backgrounds and drug interactions. 
In conclusion, in the present study, a novel UPLC-HRMS procedure for the measurement of CsA in human whole blood was successfully developed and validated. The positive bias of the CMIA for renal transplant patients when compared to the novel UPLC-HRMS application should be further evaluated by incorporating more clinical information.

\section{Acknowledgements}

The authors would like to thank Professor Fei Shang (Analyst and Test Center, Beijing University of Chemical Technology, Beijing, China) for helping to establish the UPLC-HRMS method.

\section{Funding}

This work was supported by Natural Science Foundation of Beijing Municipality (grant no. 7192190), Wu Jieping Medical Foundation (grant no. 320.6750.2020-04-3), the National Natural Science Foundation of China (grant no. 81503175) and the National key R\&D plan (grant no. 2020YFC2005504).

\section{Availability of data and materials}

The datasets used and/or analyzed during the current study are available from the corresponding author on reasonable request.

\section{Authors' contributions}

XW and WQ was responsible for the study conception and performed the statistical analysis, manuscript preparation and editing. WC performed data acquisition. HL performed the experiments. DZ and XZ performed the data analysis. PL designed the study and revised the manuscript. All authors read and approved the final manuscript.

\section{Ethics approval and consent to participate}

The study was approved by China-Japan Friendship Hospital (Beijing, China; no. 2019-143-K99). Written informed consent was obtained from all patients.

\section{Patient consent for publication}

Not applicable.

\section{Competing interests}

The authors declare that they have no competing interests.

\section{References}

1. Hajkova M, Jaburek F, Porubska B, Bohacova P, Holan V and Krulova M: Cyclosporine a promotes the therapeutic effect of mesenchymal stem cells on transplantation reaction. Clin Sci (Lond) 133: 2143-2157, 2019.

2. Kurata Y, Kuzuya T, Miwa Y, Iwasaki K, Haneda M, Amioka K, Yamada K, Watarai Y, Katayama A, Uchida K and Kobayashi T: Clinical relevance of post-transplant pharmacodynamic analysis of cyclosporine in renal transplantation. Int Immunopharmacol 22: 384-391, 2014.
3. Hryniewiecka E, Żegarska J, Żochowska D, Jaźwiec R, Borowiec A, Samborowska E, Tszyrsznic W, Dadlez M and Paczek L: Hydroxylated, hydroxymethylated, dihydroxylated, and trihydroxylated cyclosporine metabolites can be nephrotoxic in kidney transplant recipients. Transplant Proc 48: 1551-1555, 2016.

4. Seger C, Shipkova M, Christians U, Billaud EM, Wang P, Holt DW, Brunet M, Kunicki PK, Pawiński T, Langman LJ, et al: Assuring the proper analytical performance of measurement procedures for immunosuppressive drug concentrations in clinical practice: Recommendations of the international association of therapeutic drug monitoring and clinical toxicology immunosuppressive drug scientific committee. Ther Drug Monit 38: 170-189, 2016.

5. Mika A and Stepnowski P: Current methods of the analysis of immunosuppressive agents in clinical materials: A review. J Pharm Biomed Anal 127: 207-231, 2016.

6. Brate EM, Finley DM, Grote J, Holets-McCormack S, Ozaeta PF, Pacenti D, Peart JE, Piktel RE, Ramsay CS, Rupprecht KR, et al: Development of an abbott ARCHITECT cyclosporine immunoassay without metabolite cross-reactivity. Clin Biochem 43: 1152-1157, 2010.

7. Stettin M, Halwachs-Baumann G, Genser B, Frühwirth F, März W and Khoschsorur GA: Determination of cyclosporine a in whole blood: Comparison of a chromatographic method with three different immunological methods. Talanta 69: 1100-1105, 2006.

8. Henry H, Sobhi HR, Scheibner O, Bromirski M, Nimkar SB and Rochat B: Comparison between a high-resolution single-stage orbitrap and a triple quadrupole mass spectrometer for quantitative analyses of drugs. Rapid Commun Mass Spectrom 26: 499-509, 2012.

9. Bruns K, Mönnikes R and Lackner KJ: Quantitative determination of four immunosuppressants by high resolution mass spectrometry (HRMS). Clin Chem Lab Med 54: 1193-1200, 2016.

10. Wagmann L, Hemmer S, Caspar AT and Meyer MR: Method development for quantitative determination of seven statins including four active metabolites by means of high-resolution tandem mass spectrometry applicable for adherence testing and therapeutic drug monitoring. Clin Chem Lab Med 58: 664-672, 2020.

11. Du F, Liu T, Shen T, Zhu F and Xing J: Qualitative-(semi) quantitative data acquisition of artemisinin and its metabolites in rat plasma using an LTQ/Orbitrap mass spectrometer. J Mass Spectrom 47: 246-252, 2012.

12. Shipkova M and Svinarov D: LC-MS/MS as a tool for TDM services: Where are we? Clin Biochem 49: 1009-1023, 2016.

13. Jannetto PJ and Fitzgerald RL: Effective use of mass spectrometry in the clinical laboratory. Clin Chem 62: 92-98, 2016.

14. Food and Drug Administration (FDA): Bioanalytical Method Validation Guidance for Industry, 2018. https://www.fda.gov/files/ drugs/published/Bioanalytical-Method-Validation-Guidance-forIndustry.pdf.

15. Passing $\mathrm{H}$ and Bablok $\mathrm{W}$ : A new biometrical procedure for testing the equality of measurements from two different analytical methods. Application of linear regression procedures for method comparison studies in clinical chemistry. Part I J Clin Chem Clin Biochem 21: 709-720, 1983.

16. Bland JM and Altman DG: Statistical methods for assessin agreement between two methods of clinical measurement. Lancet 1 : 307-310, 1986.

17. Oellerich M, Armstrong VW, Kahan B, Shaw L, Holt DW, Yatscoff R, Lindholm A, Halloran P, Gallicano K, Wonigeit K, et al: Lake louise consensus conference on cyclosporin monitoring in organ transplantation: Report of the consensus panel. Ther Drug Monit 17: 642-654, 1995.

18. Oellerich M, Armstrong VW, Schütz E and Shaw LM: Therapeutic drug monitoring of cyclosporine and tacrolimus. Update on lake louise consensus conference on cyclosporin and tacrolimus. Clin Biochem 31: 309-316, 1998.

19. Hamwi A, Salomon A, Steinbrugger R, Fritzer-Szekeres M, Jäger W and Szekeres T: Cyclosporine metabolism in patients after kidney, bone marrow, heart-lung, and liver transplantation in the early and late posttransplant periods. Am J Clin Pathol 114: 536-543, 2000.

20. Wang CP, Burckart GJ, Ptachcinski RJ, Venkataramanan R, Schwinghammer T, Hakala T, Griffith B, Hardesty R, Shadduck R, Knapp J, et al: Cyclosporine metabolite concentrations in the blood of liver, heart, kidney, and bone marrow transplant patients. Transplant Proc 20 (Suppl 2): 591-596, 1988. 
21. Vyzantiadis T, Belechri AM, Memmos D, Axiotou M, Vyzantiadis A and Papadimitriou M: Cyclosporine and its metabolites before and $2 \mathrm{~h}$ post-dose: Comparative measurements of a monoclonal and a polyclonal immunoassay. Clin Transplant 17: 231-233, 2003.

22. Perry RH, Cooks RG and Noll RJ: Orbitrap mass spectrometry: Instrumentation, ion motion and applications. Mass Spectrom Rev 27: 661-699, 2008.

23. Mei S, Wang J, Chen D, Zhu L, Zhao M, Hu X, Yang L and Zhao Z: Ultra-high performance liquid chromatography tandem mass spectrometry for cyclosporine analysis in human whole blood and comparison with an antibody-conjugated magnetic immunoassay. Ther Drug Monit 40: 69-75, 2018.

24. Mei S, Wang J, Chen D, Zhu L, Zhao M, Tian X, Hu X and Zhao Z: Simultaneous determination of cyclosporine and tacrolimus in human whole blood by ultra-high performance liquid chromatography tandem mass spectrometry and comparison with a chemiluminescence microparticle immunoassay. J Chromatogr B Analyt Technol Biomed Life Sci 1087-1088: 36-42, 2018

25. Li W, Li R, Liu H, Guo X, Shaikh AS, Li P, Wang B, Guo R and Zhang R: A comparison of liquid chromatography-tandem mass spectrometry (LC-MS/MS) and enzyme-multiplied immunoassay technique (EMIT) for the determination of the cyclosporin A concentration in whole blood from Chinese patients. Biosci Trend 11: 475-482, 2017.

26. Tszyrsznic W, Borowiec A, Pawlowska E, Jazwiec R, Zochowska D, Bartlomiejczyk I, Zegarska J, Paczek L and Dadlez M: Two rapid ultra performance liquid chromatography/tandem mass spectrometry (UPLC/MS/MS) methods with common sample pretreatment for therapeutic drug monitoring of immunosuppressants compared to immunoassay. J Chromatogr B Analyt Technol Biomed Life Sci 928: 9-15, 2013.
27. Sadeg N, Pham-Huy C, Rucay P, Righenzi S, Halle-Pannenko O, Claude JR, Bismuth H and Duc HT: In vitro and in vivo comparative studies on immunosuppressive properties of cyclosporines A, C, D and metabolites M1, M17 and M21. Immunopharmacol Immunotoxicol 15: 163-177, 1993.

28. Yatscoff RW, Rosano TG and Bowers LD: The clinical significance of cyclosporine metabolites. Clin Biochem 24: 23-35, 1991.

29. Ansermot N, Fathi M, Veuthey JL, Desmeules J, Rudaz S and Hochstrasser D: Quantification of cyclosporine and tacrolimus in whole blood. Comparison of liquid chromatography-electrospray mass spectrometry with the enzyme multiplied immunoassay technique. Clin Biochem 41: 910-913, 2008.

30. Ko DH, Cho EJ, Lee W, Chun S and Min WK: Accuracy evaluation of Roche and Siemens tacrolimus and cyclosporine assays in comparison with liquid chromatography-tandem mass spectrometry. Scand J Clin Lab Invest 78: 431-438, 2018.

31. Cangemi G, Barco S, Bonifazio P, Maffia A, Agazzi A and Melioli G: Comparison of antibody-conjugated magnetic immunoassay and liquid chromatography-tandem mass spectrometry for the measurement of cyclosporine and tacrolimus in whole blood. Int J Immunopathol Pharmacol 26: 419-426, 2013.

32. Lee Y: Comparison between ultra-performance liquid chromatography with tandem mass spectrometry and a chemiluminescence immunoassay in the determination of cyclosporin A and tacrolimus levels in whole blood. Exp Ther Med 6: 1535-1539, 2013.

This work is licensed under a Creative Commons Attribution-NonCommercial-NoDerivatives 4.0 International (CC BY-NC-ND 4.0) License. 